\title{
Integrating facilitative teaching in design based course
}

\begin{abstract}
Introducing a highly technical multidisciplinary design problem to first year students can be a daunting task. First, their knowledge experience revolves only about basic mathematics, science and its application to technological problem. If mishandled, exposing them to complex design process at this stage can pose a disastrous effect. The intent of this paper is to share the facilitative teaching approach performed onto the first year students at the Department of Chemical \& Environmental Engineering, UPM. This facilitative teaching approach is specifically adopted in the Material Balances courses $(2+1)$ where a multidisciplinary conceptual design project is embedded as part of its content. The teaching team is responsible to plan and design instruction, assessment and evaluation materials, coach team during design activities by providing assistance and on time feedback, and maintain teams' vibrancy. A highly committed teaching team is required to materialize this approach because the efforts and time spent to conduct the activities for learning is not equivalent in number to the given credit loading. Furthermore, time and energy for other activities related to key performance indicators are usually sacrificed. However, excellent students' engagement, enthusiasm and participation to learn indicate that the efforts and time were worth spent.
\end{abstract}

Keyword: Facilitative teaching; Design course; First year 